\title{
AN ATLAS-BASED DEEP BRAIN STRUCTURE SEGMENTATION METHOD: FROM COARSE POSITIONING TO FINE SHAPING
}

\author{
Yishan LUO and Albert C.S. CHUNG \\ Lo Kwee-Seong Medical Analysis Laboratory, \\ Department of Computer Science and Engineering, \\ The Hong Kong University of Science and Technology, Hong Kong
}

\begin{abstract}
Segmentation of deep brain structures is a challenging task for MRI images due to blurry structure boundaries, small object size and irregular shapes. In this paper, we present a new atlas-based segmentation method. It first uses a prior spatial dependency tree to constrain the relative positions between different deep brain structures and determine an optimal sequence for the structureby-structure segmentation. After positioning the structures, the segmentation result is further fine tuned by a non-rigid registration procedure between the atlas image and the target image using the histogram of the gradient magnitudes lying on the structure boundaries. The proposed method has been applied on a publicly available MRI brain database and can achieve comparatively high segmentation accuracy.
\end{abstract}

Index Terms - deep brain structure, segmentation

\section{INTRODUCTION}

The segmentation of brain structures in magnetic resonance imaging (MRI) is crucial in neuroscience with many applications, such as mapping of functional activation onto brain anatomy, the study of brain development and so on. However, obtaining accurate segmentation results is challenging because of the random noise, inhomogeneity and weak boundaries in medical images. Among various brain structures, deep brain structures such as the caudate nucleus $(\mathrm{CN})$, the putamen $(\mathrm{Pu})$ and the thalamus (Th) play critical roles in human brain functioning. However, these deep brain structures are relatively harder to be segmented due to excessively blurred boundaries and small sizes.

Atlas-based segmentation algorithms have been successfully applied in this area $[1,2,3,4]$. Atlas-based method is trying to find a geometric transformation from the pre-labeled atlas image to the target image and propagate the labels of the atlas with the same transformation to label the target image.
In this paper, we propose a novel atlas-based segmentation method for deep brain structures, which is grounded on the following two observations. First, although the same structure of different subjects can have entirely different positions, there exist some rules in the relative positions between different structures within one subject. Second, the histogram of the gradient magnitudes on the deep brain structure boundary follows a right-skewed distribution. Based on these observations, our method is proposed with the following distinct features. (1) The deep brain structures are segmented in sequence, with a prior spatial dependency tree to constrain their inter-relations and determine the segmentation order. Different with most of the brain segmentation methods which treat the brain volume as a whole, we focus on one specific structure of interest each time. This can not only greatly reduce the computational cost, but also avoid deforming different structures in a similar way. (2) Some statistic information describing the shape of gradient magnitude histogram is embedded into the energy function for refining the segmentation result. The features of the method endow it with well-positioned and automatic initialization, the ability to handle blurry boundary and lessened training work for atlas building.

\section{METHODOLOGY}

The entire algorithm can be divided into two steps. The first step is to locate each structure with linear transformation. The spatial dependency relations between the deep brain structures are exploited to determine an optimal structure segmentation sequence and constrain the positions of these structures. The second step is to further refine the segmentation result using a non-rigid image registration procedure.

\subsection{Prior Spatial Dependency Tree}

Considering only intensity information of the brain image is difficult to segment the deep brain structures, as many structures have similar intensity profiles over the entire volume. With this concern, spatial information 
becomes crucial in differentiating structures. Through learning from the brain database and supported by the structure descriptions in the anatomy literatures, some spatial dependencies of these structures can be deduced.

First of all, the ventricles (Ven) are relatively easier to be segmented in the brain scans as it has relatively sharp boundary and more differentiable intensity information. Furthermore, the positions of deep brain structures have some spatial connections with Ven. Therefore, Ven will be segmented first. With the segmented Ven, the relative positions of the $\mathrm{CN}, \mathrm{Pu}$ and $\mathrm{Th}$ are subsequently constrained. The inter-structure relations can be deduced from a widely used MRI brain image database IBSR_V2.0 ${ }^{1}$. This database includes 18 subjects' T1-weight volumetric images together with their manual segmentations, which have been positionally normalized into the Talairach orientation. Half of them (9 subjects) were used for finding the spatial relation rules. The other half of the image volumes were used for evaluating the algorithm.

With the segmentation ground truth of the 9 subjects, we can calculate centers of mass of the following structures: Ven, $\mathrm{CN}, \mathrm{Pu}$ and Th. With the dimension definition of the Talairach space that $X$ is the Left/Right dimension, $Y$ is the Posterior/Anterior dimension and $Z$ is the Inferior/Superior dimension, the centers of mass calculated from the 9 data sets are shown in X-Y, Z-X, $\mathrm{Z}-\mathrm{Y}$ planes in Fig.1. The following rules can be easily inferred accordingly, and some of them are supported in the anatomy literatures.

- Along the X dimension, the distance between LPu and $\mathrm{RPu}$ is the largest among the four structures.

- The Y coordinate relationship between the four structures is $\mathrm{CN}<\mathrm{Pu}<\{$ Ven, $\mathrm{Th}\}$.

- The Z coordinate relationship between the four structures is $\mathrm{Pu}<\mathrm{Th}<\{\mathrm{CN}, \mathrm{Ven}\}$.

A tree model is then built based on the spatial dependency, as depicted in Fig.2. Generally speaking, the higher level object is the parent (indicated by solid arrow) or ancestor (indicated by dashed arrow) of the lower level objects. Thus the positions of the lower level objects will be constrained by their ancestors. The tree model also determines the segmentation sequence, which is proceeded from the top to the bottom. The lower level structures will be segmented after all their ancestors have been segmented, as such their positions can be fully constrained adhere to the rules.

\subsection{Energy Function}

The energy function for the first step is formulated through the maximum a posteriori (MAP) method.

\footnotetext{
${ }^{1}$ http://www.cma.mgh.harvard.edu/ibsr/
}

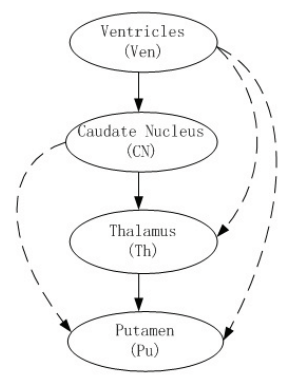

Fig. 2. The tree model for the structure-by-structure segmentation. Both the parent-child relationship (the solid arrow) and the ancestor-descendant relationship (the dashed arrow) are shown.

Suppose $L \in\{V e n, C N, T h, P u\}$ is the structure label, $C \in\left\{C_{V e n}, C_{C N}, C_{T h}, C_{P u}\right\}$ is the center of mass for each structure, and the observed intensity of the target image is represented by $Y$. According to the Bayes' theorem, we have

$$
P(L, C \mid Y) \propto P(Y \mid L, C) P(L, C),
$$

$P(Y \mid L, C)$ is the likelihood of the observed data given the structure label and the center of mass. The optimal segmentation results can be obtained by maximizing the posteriori probability. Also given the segmentation label $L$, the observed intensity $Y$ should be independent of position of the mass center $C$. Then the optimal estimator of $\mathrm{L}$ and $\mathrm{C}$ can be obtained by,

$$
\left(L^{*}, C^{*}\right)=\arg \max [P(Y \mid L) P(L, C)] .
$$

Taking logarithm of the product and transforming maximization to minimization yield the following energy function to be minimized:

$$
\arg \min E_{i}=\arg \min [-\ln (P(Y \mid L))-\ln (P(L, C))] \text {, }
$$
where $i=V e n, C N, T h, P u$. By using functions with the image property dependencies implied by the probabilities, similar as in [5], Equation 3 becomes

$\arg \min E_{i}=\arg \min \left[M_{r e g}(Y, L)+M_{\text {pos }}(L, C)\right]$.

The first term is measured by,

$$
M_{r e g}(Y, L)=\frac{1}{V} \sum_{L}|y-\bar{y}|
$$

where $y$ is the intensity value of each voxel in the structure $L, \bar{y}$ is the average intensity within $L$ and $V$ is the volume of the structure.

The second term is defined according to the prior spatial dependency rules. Here the mass centers of Ven, $\mathrm{CN}, \mathrm{Th}, \mathrm{Pu}$ are, respectively, represented by $\left(v_{x}, v_{y}, v_{z}\right)$, $\left(c_{x}, c_{y}, c_{z}\right),\left(t_{x}, t_{y}, t_{z}\right),\left(p_{x}, p_{y}, p_{z}\right)$.

- LVen (RVen): Root object, no spatial constraint.

- $\mathrm{LCN}(\mathrm{RCN})$ : The child object of LVen (RVen), with a constraint on the $Y$ dimension.

$$
M_{\text {pos }}(L, C)=\left|c_{y}-v_{y}\right| H\left(c_{y}-v_{y}\right),
$$

$\mathrm{H}($.$) is the Heaviside step function.$ 

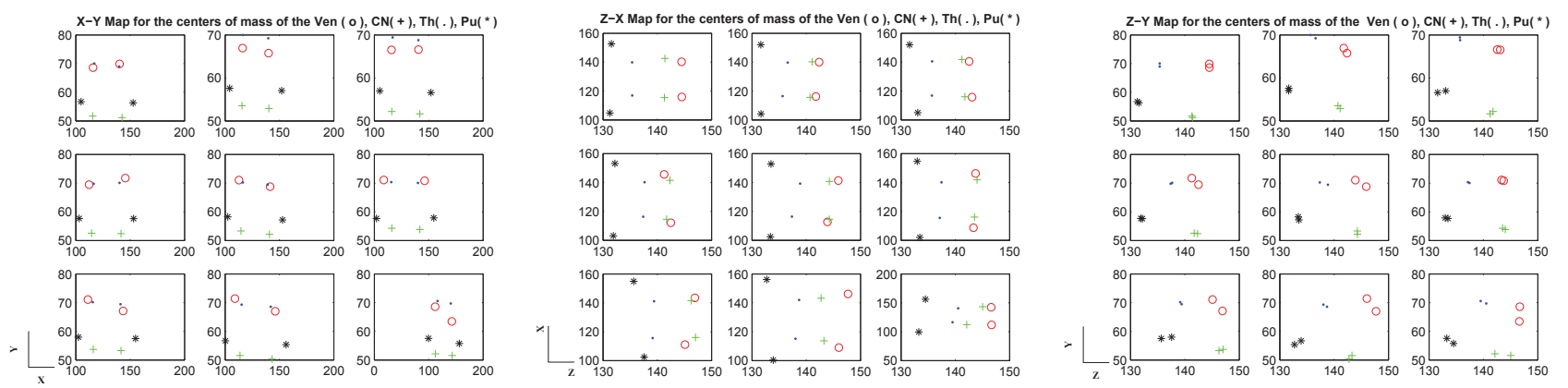

Fig. 1. (Colour Images) Positions of the centers of mass for the left Ven(LVen) and right Ven(RVen) (red 'o'), left CN(LCN) and right $\mathrm{CN}(\mathrm{RCN})$ (green '+' ), left $\mathrm{Th}(\mathrm{LTh})$ and right $\mathrm{Th}(\mathrm{RTh})$ (blue '.') and left $\mathrm{Pu}(\mathrm{LPu})$ and right $\mathrm{Pu}(\mathrm{RPu})(\mathrm{black}$ '*') are shown in X-Y, Z-X, Z-Y planes. The 9 sub-figures in each plane represent 9 data sets. The unit of axis is voxel.

- LTh (RTh): The child object of LCN (RCN), thus descendant of LVen (RVen), with constraints on $Y$ and $Z$ dimensions.

$$
\begin{aligned}
M_{p o s}(L, C) & =\left[\left|c_{y}-t_{y}\right| H\left(c_{y}-t_{y}\right)+\left|t_{z}-c_{z}\right| H\left(t_{z}-c_{z}\right)\right. \\
& \left.+\left|t_{z}-v_{z}\right| H\left(t_{z}-v_{z}\right)\right] / n .
\end{aligned}
$$

where $n$ is a normalization factor. It equals to the number of nonzero elements among the three summed elements in the numerator.

- LPu (RPu): The child object LTh (RTh), thus descendant of LVen (RVen), LCN (RCN), with constraints on the $X, Y$ and $Z$ dimensions.

For LPu:

$$
\begin{aligned}
M_{\text {pos }}(L, C) & =\left[\left|p_{x}-v_{x}\right| H\left(p_{x}-v_{x}\right)+\left|p_{x}-c_{x}\right| H\left(p_{x}-c_{x}\right)\right. \\
& +\left|p_{x}-t_{x}\right| H\left(p_{x}-t_{x}\right)+\left|c_{y}-p_{y}\right| H\left(c_{y}-p_{y}\right) \\
& +\left|p_{y}-v_{y}\right| H\left(p_{y}-v_{y}\right)+\left|p_{y}-t_{y}\right| H\left(p_{y}-t_{y}\right) \\
& \left.+\left|p_{z}-t_{z}\right| H\left(p_{z}-t_{z}\right)\right] / n .
\end{aligned}
$$

and $\mathrm{RPu}$ :

$$
\begin{aligned}
M_{\text {pos }}(L, C) & =\left[\left|v_{x}-p_{x}\right| H\left(v_{x}-p_{x}\right)+\left|c_{x}-p_{x}\right| H\left(c_{x}-p_{x}\right)\right. \\
& +\left|t_{x}-p_{x}\right| H\left(t_{x}-p_{x}\right)+\left|c_{y}-p_{y}\right| H\left(c_{y}-p_{y}\right) \\
& +\left|p_{y}-v_{y}\right| H\left(p_{y}-v_{y}\right)+\left|p_{y}-t_{y}\right| H\left(p_{y}-t_{y}\right) \\
& \left.+\left|p_{z}-t_{z}\right| H\left(p_{z}-t_{z}\right)\right] / n .
\end{aligned}
$$

With the defined energy function, the whole positioning step begins with the segmentation ground truth for each structure of the atlas image, which serves as an automatic initialization. The initial structure model is deformed within the target image by the affine transform to minimize the energy function in Equation 4. As the segmentation is conducted structure-by-structure, each structure will have a separate affine transformation.

\subsection{Refinement With Gradient Information}

After obtaining the coarse segmentation with the affine transformation, a non-rigid registration based segmentation with B-spline transform is followed to refine the segmentation result. In this step, the gradient information on the structure boundaries is utilized for refinement.
Similar with Equation 1, we have,

$$
P(L, G \mid X, Y) \propto P(X, Y \mid L, G) P(L, G),
$$

where $X$ and $Y$ are respectively the atlas image and the target image, $G$ represents the gradient information on the structure boundary. $L$ and $G$ are casually independent w.r.t. $X$ and $Y$, thus

$$
P(X, Y \mid L, G)=P(X, Y \mid L) P(X, Y \mid G) .
$$

Similar as Section 2.1, the energy function for the refinement step is formulated as,

$$
\begin{aligned}
\arg \min E & =\arg \min \left[M_{\text {reg }}(X, Y, L)\right. \\
& \left.+M_{\text {bound }}(X, Y, G)+M_{\text {bound }}(L, G)\right] .
\end{aligned}
$$

The first term is expressed by

$$
M_{\text {reg }}(X, Y, L)=\frac{1}{V} \sum_{L}|y-\bar{x}|,
$$

where $\bar{x}$ is the average intensity within the structure $L$ of the atlas image. It prefers a homogeneous structure region within the target image with similar intensity distribution as the same structure of the atlas image.

The second term of Equation 12 is given by,

$$
M_{\text {bound }}(X, Y, G)=\left|\bar{G}_{x}-\bar{G}_{y}\right|,
$$

where $\bar{G}_{x}$ and $\bar{G}_{y}$ are the average gradient magnitudes on the structure boundaries of the atlas and target images. It searches a region within the target image with similar boundary information as the atlas image.

The third term is the gradient information given the structure labels. It is observed from the IBSR database that, the histogram of gradient magnitudes along the structure boundary follows a right-skewed shape as depicted in Fig.3. The descriptive statistics, i.e., mean, median and mode, can be utilized to describe the rightskewed shape of a distribution. They obey the rule that if the distribution is skewed to the right, the mode is less than the median and the median is less than the mean. Therefore, the third term of the energy function is formulated as,

$$
\begin{aligned}
& M_{\text {bound }}(L, G)=[|m 1-m 2| H(m 1-m 2) \\
& \quad+|m 2-m 3| H(m 2-m 3)+|m 1-m 3| H(m 1-m 3)] / n,
\end{aligned}
$$



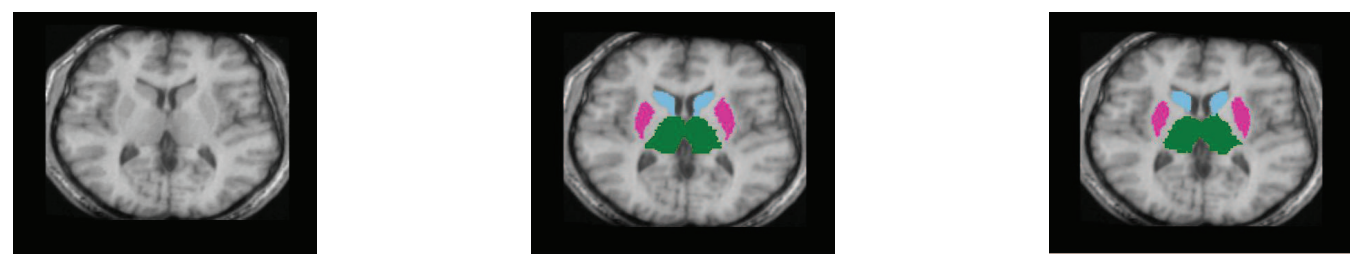

Fig. 4. (Colour Images)Segmentation result of the CN (blue), Th (green) and Pu (purple) from one 3D example. Slices are taken from the $3 \mathrm{D}$ volume. From the left to the right are MRI scan, segmentation ground truth and our segmentation result.

where $m 1, m 2$ and $m 3$ are respectively the mode, median and mean of the gradient magnitudes on the structure boundary. Descriptive statistics of the gradient histogram on structure boundaries are utilized to refine the segmentation results. It can alleviate the dependence on strong edges. With the coarse segmentation result in the first step as the starting point, the non-rigid registration with B-spline based free-form deformation (FFD) is performed between the atlas image and the target image. Through minimizing the energy function (12), the optimal transformation relating the atlas image and the target image can be determined. Then the labels of the atlas image will be propagated with the obtained transformation to label the target images.

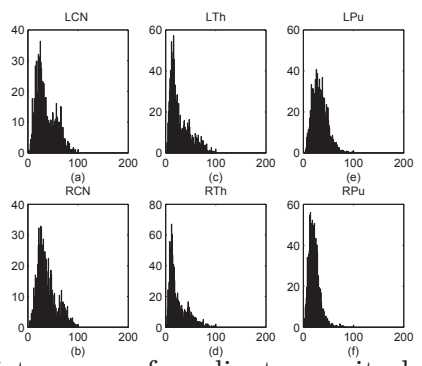

Fig. 3. The histograms of gradient magnitude on the boundaries of the deep structures. ((a) LCN (b) RCN (c) LTh (d) $\mathrm{RTh}(\mathrm{e}) \mathrm{LPu}(\mathrm{f}) \mathrm{RPu}$ )

\section{EXPERIMENTAL RESULTS}

In the experiments, the proposed method was applied on the brain database from IBSR. Among the $18 \mathrm{MR}$ scans, 9 data sets were used to deduce the spatial dependency rules, the other 9 data sets were served as testing data. In the testing set, a randomly chosen data set was served as the atlas image with its segmentation ground truth as atlas model, other data sets were treated as the target images to be segmented. The L-BFGS optimizer is utilized to optimize the energy functions in Equations 4 and 12. We implemented the algorithm with Matlab $\mathrm{v} 7.8 .0$, the computation time for each data pair is around 1 to 2 hours on a $3.16 \mathrm{GHz} \mathrm{CPU}$ with $3 \mathrm{~GB}$ memory. The segmentation results were compared with the expert segmentations available from the IBSR database. The extent of volume overlapping between experimental results and the ground truth is measured by the Dice similarity coefficient (DSC) defined as $\frac{2\|A \cap B\|}{\|A\|+\|B\|}$, where $\mathrm{A}$ and $\mathrm{B}$ are the compared data sets. In Table 1, we compare the DSCs results of our methods with other related works $[3,6]$, which are the state-of-the-art works using the same database as ours. As shown in Table 1, our method shows superior performance compared with other methods. An example of segmentation result is also shown in Fig.4. Slices obtained from a 3D volume are presented, together with its segmentation ground truth and our segmentation result.

Table 1. The DSC comparison between the proposed method and other related works.

\begin{tabular}{c|c|c|c}
\hline Methods & Th & $\mathrm{Pu}$ & $\mathrm{CN}$ \\
\hline Proposed & 0.84 & 0.80 & 0.78 \\
Ref. [3] & 0.60 & 0.49 & 0.54 \\
Ref. [6] & 0.77 & 0.70 & 0.65 \\
\hline
\end{tabular}

\section{CONCLUSION}

In this paper, a novel segmentation method for deep brain structures is presented. The proposed method can achieve higher segmentation accuracy than the state-ofthe-art methods. Our method is preferable for the following reasons: (1) No manual initialization is neede. (2) The spatial dependency rules can be easily inferred; (3) Ability to handle structures with blurry boundaries; (4) Lessened burden on training for building atlas as compared with $[2,4]$. These atlas-based methods usually need sufficient training to build an atlas so as to obtain improved performance. Comparatively, our method, which only uses one randomly chosen atlas, can also achieve competitive result.

\section{REFERENCES}

[1] Bouix S. Pohl K., "Nakamura m.:a hierarchical algorithm for $\mathrm{mr}$ brain image parcellation," TMI, vol. 26, pp. 1201$12,2007$.

[2] Wang L. Khan A. and Beg M, "Freesurfer-initiated fullyautomated subcortical brain segmentation in mri using lddmm," NeuroImage, vol. 41, pp. 735-46, 2008.

[3] Shattuck D.W. Joshi A.A. and Thompson P.M., "Surfaceconstrained volumetric brain registration using harmonic mappings," TMI, vol. 26, pp. 1657-69, 2007.

[4] Powell S., "Registration and machine learning-based automated segmentation of subcortical and cerebellar brain structuress," NeuroImage, vol. 39, pp. 238-47, 2008.

[5] Staib L. H. Chakraborty A. and Duncan J. S., "Deformable boundary finding in medical images by integrating gradient and region information," TMI, vol. 15(6), pp. 859-870, 1996.

[6] Barillot C. Ciofolo C., "Brain segmentation with competitive level sets and fuzzy control," IPMI, vol. 3565, pp. 333-44, 2005. 